\title{
Determination of Settings of a Tilted Head- Cutter for Generation of Hypoid and Spiral Bevel Gears
}

F.L. Litvin and Y. Zhang

University of Illinois at Chicago

Chicago, Illinois

and

M. Lundy and C. Heine

Dana Corporation

Fort Wayne, Indiana

(AASA-CR-182138) DETEEMINAIICN CF SET TINGS

CF A TILTED HEAL-CETIER FCE EENEEATION OF

EYPOID AND SPIERI EEVEI GEAES. Final Beport

(1)linois Univ:) $27 \mathrm{~F}$

CSCL 1.31
$N 88-2.49 .76$

Unclas

\section{G3/37 0148131}

June 1988

Prepared for

Lewis Research Center

Under Grant NAG3-783 


\title{
DETERMINATION OF SETTINGS OF A TILTED HEAD-CUTTER POR GENERATION OF HYPOID AND SPIRAL BEVEL GEARS
}

\author{
F.L. Litvin and $Y$. Zhang \\ University of Illinois at Chicago \\ Chicago, Illinois 60688 \\ and \\ M. Lundy and C. Heine \\ Dana Corporation \\ Fort Wayne, Indiana
}

\begin{abstract}
Kinematics of Gleason mechanisms of hypoid and spiral bevel cutting machines is considered. These mechanisms are designated to install the position and tilt the head cutter. The tilt of the head cutter with standard blades provides the required pressure angle. The authors have developed the matrix presentation of kinematics of these mechanisms and basic equations for the required settings. An example is presented based on the developed computation procedure.
\end{abstract}

\section{Introduction}

The synthesis of hypoid and spiral bevel gears is an important problem that has been a subject of intensive research by Gleason engineers [1] and Litvin et al. [2]. Gleason engineers have designed machines to cut and grind hypoid and spiral bevel gears that are widely used in the industry. An ingenious mechanism is used in the Gleason gear cutting machines. This mechanism provides the required pinion pressure angle by tilting the head-cutter and thereby reducing the number of different cutter blades. A short description of the tilt head-cutter mechanism has been given by 
Lehmann [3] but the basic equations that are necessary for the settings have not been presented. The purpose of this paper is to investigate the kinematics of the tilt-mechanism and to derive the basic equations for the installment of the machinetool settings.

Henceforth we will use two coordinate systems, $s_{k}^{(i)}$ and $s_{k}^{(j)}$, that are rigidly connected to link $k$. However, only one coordinate system, $s_{0}^{(i)}$, is rigidly connected to the frame of the cutting machine and $z_{0}^{(i)}$ is the axis of rotation of the cradle (Fig. 1.1,a). The head-cutter is pointed to the observer. It is assumed that vectors $s$ and $c$ are known and they are represented in $s_{0}^{(i)}$. These vectors have been already determined at the stage of local synthesis [2]. Vector $s=0_{0}^{(i)} 0_{1}^{(i)}$ is located in the machine plane $z_{0}^{(i)}=0$ and is represented by the row matrix (Fig. $1.1, b)$

$$
\underline{s}=\left[\begin{array}{lll}
s \cos q & -\mathrm{s} \text { sinq. }
\end{array}\right]
$$

where $s=\left|\overline{0_{0}^{(i)} o_{1}^{(i)}}\right|$ and $q$ determine the magnitude and orientation of vector $s$. Vector $s$ is the unit vector of the axis of the tilted head cutter and is represented in $s_{0}^{(i)}$ by the following row matrix (Fig. 1.1,b)

$$
\left[\underline{c}_{0}\right]=\cdot\left[\begin{array}{lll}
\cos p & -\operatorname{sinp} \quad \cos \mu
\end{array}\right]
$$

Here: $p$ is the angle that determines the orientation of vector $c^{*}$, the projection of vector $\stackrel{c}{\sim}$ on the machine plane; $\mu$ is the angle that is formed between the axis of the cradle and the axis of the tilted head-cutter. The orientation of vector $\subseteq$ must satisfy the following requirements: (i) it must be perpendicular to plane $P$ that is tangent to the root cone of the generated gear (see section A-A in Fig. $1.1, \mathrm{~b}$ ) and (ii) the unit normal to the tilted cone of the head cutter must coincide with the 
unit normal $\underset{\sim}{\mathrm{n}}$ to the surfaces of the mating gears (see section B-B in Fig. $1.1, \mathrm{~b})$. Due to the tilt of the head-cutter it becomes possible to use blades with the same shape angle $\psi_{C}$ for various orientation of the surface unit normal $\underline{n}$ (section A-A in Fig. $1,1, b)$.

The sketch of the mechanism for the installment of the machine-tool settings is shown in Fig. 1.2. The cradle (1) can be turned about the a-axis of the cutting machine. The cradle carries the so-called eccentric (2) that can be turned about the b-axis that is mounted on the cradle. The eccentric carries the swivel ( 3 ) that is provided with two joints whose axes are intersected and form angle $\varepsilon$. The swivel can be turned about the c-axis of the eccentric. The cutter spindle (4) is a link that is also provided with two joints, $d$ and $e$, whose axes form the same angle $\varepsilon$ as the joints of the swive1. The cutter spindle carries the hear-cutter that rotates about the e-axis in the process of gear generation. Plane $P$ of the blades passes through the point of intersection of axes $d$ and $e$. The rotation of the head-cutter provides the desired velocity of cutting. However, when deriving the equations for the installment of machine-tool setting, we may assume that the head-cutter and the cutter spindle are rigidly connected. The tilt mechanism may be also represented as shown in Fig.1.3. The swivel and the head-cutter spindle are interconnected by the wedge. The relative motion of the cutter spindle with respect to the swivel is rotation about the d-axis that is perpendicular to the wedge.

It will be shown in section 3 of the paper that the required magnitude and orientation of position-vector $s$ (Fig. $1.1, b$ ) is provided by the turning of the cradle and the eccentric. The required orientation of the unit vectors $\subseteq$ are provided with the turn of the swivel and the cutter spindle. 


\section{Basic Kinematic Equations}

The basic kinematic equations represent the machine-tool settings in terms of given parameters of vectors $s$ and $\subseteq$ (Fig. 1.1). The derivation of equations for settings is based on matrix representation of the coordinate transformation.

The mechanism for the installment of the machine-tool settings is shown in Fig: 1.2. The schematic of the mechanism and the applied coordinate systems are shown in Fig. 2.1. We consider that the cradle (1), the eccentric (2), the swivel (3) and the cutter spindle (4) are provided with two rigidly connected coordinate systems $S_{k}^{(i)}$ and $S_{k}^{(j)}(k=1,2,3,4)$. We will use for the coordinate transformation matrices of two types, $G$ and $M$, that describe the link geometry, and the relative motion of interconnected links, respectively. The coordinate transformation from link 4 to link 0 is represented as follows

$$
\begin{aligned}
& S_{0}^{(i)}+S_{1}^{(j)}+S_{1}^{(i)}+S_{2}^{(j)}+S_{2}^{(i)}+S_{3}^{(j)}+S_{3}^{(i)}+S_{4}^{(j)}+S_{4}^{(i)} \\
& {\left[M_{04}^{(i i)}\right]=\left[M_{01}^{(i j)}\right]\left[G_{1}^{(j i)}\right]\left[M_{12}^{(i j)}\right]\left[G_{2}^{(j i)}\right]\left[M_{23}^{(i j)}\right]\left[G_{3}^{(j i)}\right]\left[M_{34}^{(i j)}\right]\left[G_{4}^{(j i)}\right]}
\end{aligned}
$$

Geometry matrix $\left[G^{(j i)}\right]$ for links 3 and 4 that are provided with two intersected axes of revolute joints is represented as follows (Fig. 2.2,a)

$$
\left[G_{\ell}^{(j i)}\right]=\left[\begin{array}{cccc}
\cos \varepsilon & 0 & (\bar{\mp}) \sin \varepsilon & 0 \\
0 & 1 & 0 & 0 \\
(\stackrel{ \pm}{-}) \sin \varepsilon & 0 & \cos \varepsilon & 0 \\
0 & 0 & 0 & 1
\end{array}\right] \quad(l=4,3)
$$

The upper and lower signs correspond to $\ell=4$ and $\ell=3$, respectively. Matrix $\left[G_{k}^{(j i)}\right]$ for links 2 and 1 , that are provided with two parallel axes of revolute joints is represented by (Fig. $2.2, \mathrm{~b}$ ) 


$$
\left[G_{k}^{(j i)}\right]=\left[\begin{array}{rrrr}
1 & 0 & 0 & \mp \ell \\
0 & 1 & 0 & 0 \\
0 & 0 & 1 & 0 \\
0 & 0 & 0 & 1
\end{array}\right]
$$

Here: the upper and lower signs correspond to $k=2$ and $k=1$, respectively. Matrix $\left[M_{n m}^{(i j)}\right]$ is represented by the following equation (Fig. 2.3)

$$
\left[M_{\mathrm{nm}}^{(i j)}\right]=\left[\begin{array}{cccc}
\cos \phi_{\mathrm{nm}} & \sin \phi_{\mathrm{nm}} & 0 & 0 \\
-\sin \phi_{\mathrm{nm}} & \cos \phi_{\mathrm{nm}} & 0 & 0 \\
0 & 0 & 1 & 0 \\
0 & 0 & 0 & 1
\end{array}\right]
$$

Here: $\mathrm{n}=\mathrm{m}-1 ; \mathrm{m}=4,3,2,1$. Equations $(2.1)-(2.4)$ yield

$$
\left[M_{04}^{(i i)}\right]=\left[\begin{array}{cccc}
a_{11} & a_{12} & a_{13} & a_{14} \\
a_{21} & a_{22} & a_{23} & a_{24} \\
a_{31} & a_{32} & a_{33} & a_{34} \\
0 & 0 & 0 & 1
\end{array}\right]
$$

where

$$
\begin{aligned}
& a_{11}=\cos \tau\left(\cos ^{2} \varepsilon \cos \phi_{t}+\sin ^{2} \varepsilon\right)-\sin \tau \cos \varepsilon \sin \phi_{t} \\
& a_{12}=\cos \tau \cos \varepsilon \sin \phi_{t}+\sin \tau \cos \phi_{t} \\
& a_{13}=\frac{\cos \tau \sin 2 \varepsilon}{2}\left(1-\cos \phi_{t}\right)+\sin \tau \sin \varepsilon \sin \phi_{t} \\
& a_{14}=\ell\left[\cos \phi_{c}-\cos \left(\phi_{c}+\phi_{e}\right)\right] \\
& a_{21}=-\sin \tau\left(\cos ^{2} \varepsilon \cos \phi_{t}+\sin ^{2} \varepsilon\right)-\cos \tau \cos \varepsilon \sin \phi_{t}
\end{aligned}
$$




$$
\begin{aligned}
& a_{22}=-\sin \tau \cos \varepsilon \sin \phi_{t}+\cos \tau \cos \phi_{t} \\
& a_{23}=-\frac{\sin \tau \sin 2 \varepsilon}{2}\left(1-\cos \phi_{t}\right)+\cos \tau \sin \varepsilon \sin \phi_{t} \\
& a_{24}=-\ell\left[\sin \phi_{c}-\sin \left(\phi_{c}+\phi_{e}\right)\right] \\
& a_{31}=\frac{\sin 2 \varepsilon}{2}\left(1-\cos \phi_{t}\right) \\
& a_{32}=-\sin \varepsilon \sin \phi_{t} \\
& a_{33}=\sin ^{2} \varepsilon \cos \phi_{t}+\cos ^{2} \varepsilon \\
& a_{34}=0
\end{aligned}
$$

Here: $\tau=\left(\phi_{c}+\phi_{e}+\phi_{s}\right) ; \phi_{c}=\phi_{01}$ is the cradle angle; $\phi_{e}=\phi_{12}$ is the eccentric angle; $\phi_{S}=\phi_{23}$ is the swivel angle and $\phi_{t}=\phi_{34}$ is the cutter spindle angle. We will also need the coordinate transformation: (i) from $s_{2}^{(i)}$ to $s_{0}^{(i)}$ and (ii) from $s_{4}^{(i)}$ to $S_{2}^{(i)}$. Using the matrix equations (2.2) - (2.4), we obtain

$$
\begin{aligned}
{\left[M_{02}^{(i i)}\right] } & =\left[M_{01}^{(i j)}\right]\left[G_{1}^{(j i)}\right]\left[M_{12}^{(i j)}\right]\left[G_{2}^{(j i)}\right] \\
& =\left[\begin{array}{cccc}
\cos \left(\phi_{c}+\phi_{e}\right) & \sin \left(\phi_{c}+\phi_{e}\right) & 0 & \ell\left[\cos \phi_{c}-\cos \left(\phi_{c}+\phi_{e}\right)\right] \\
-\sin \left(\phi_{c}+\phi_{e}\right) & \cos \left(\phi_{c}+\phi_{e}\right) & 0 & -\ell\left[\sin \phi_{c}-\sin \left(\phi_{c}+\phi_{e}\right)\right] \\
0 & 0 & 1 & 0 \\
0 & 0 & 0 & 1
\end{array}\right]
\end{aligned}
$$

$$
\begin{aligned}
{\left[M_{24}^{(i i)}\right] } & =\left[M_{23}^{(i j)}\right]\left[G_{3}^{(j i)}\right]\left[M_{34}^{(i j)}\right]\left[G_{4}^{(j i)}\right] \\
& =\left[\begin{array}{cccc}
b_{11} & b_{12} & b_{13} & 0 \\
b_{21} & b_{22} & b_{23} & 0 \\
b_{31} & b_{32} & b_{33} & 0 \\
0 & 0 & 0 & 1
\end{array}\right] .
\end{aligned}
$$


Here:

$$
\begin{aligned}
& \mathrm{b}_{11}=\cos \phi_{s}\left(\cos ^{2} \varepsilon \cos \phi_{t}+\sin ^{2} \varepsilon\right)-\sin \phi_{s} \cos \varepsilon \sin \phi_{t} \\
& \mathrm{~b}_{12}=\cos \phi_{s} \cos \varepsilon \sin \phi_{t}+\sin \phi_{s} \cos \phi_{t} \\
& b_{13}=\frac{\sin 2 \varepsilon}{2}\left(1-\cos \phi_{t}\right) \cos \phi_{3}+\sin \phi_{s} \sin \varepsilon \sin \phi_{t} \\
& b_{21}=-\sin \phi_{s}\left(\cos ^{2} \varepsilon \cos \phi_{t}+\sin ^{2} \varepsilon\right)-\cos \phi_{s} \cos \varepsilon \sin \phi_{t} \\
& b_{22}=-\sin \phi_{s} \cos \varepsilon \sin \phi_{t}+\cos \phi_{s} \cos \phi_{t} \\
& b_{23}=-\frac{\sin \phi_{s} \sin 2 \varepsilon}{2}\left(1-\cos \phi_{t}\right)+\cos \phi_{s} \sin \varepsilon s i n \phi_{t} \\
& b_{31}=\frac{\sin 2 \varepsilon}{2}\left(1-\cos \phi_{t}\right) \\
& b_{32}=-\sin \varepsilon \sin \phi_{t} \\
& b_{33}=\sin { }^{2} \varepsilon \cos \phi_{t}+\cos ^{2} \varepsilon
\end{aligned}
$$

\section{Determination of Machine-Tool Settings}

\section{Determination of Cradle Angle and Eccentric Angle}

Consider that vector $s$ is given and it is represented in the machine plane $z_{0}^{(i)}=0$ as follows

$$
[s]=\left[\begin{array}{rr}
s & \cos q \\
-s & \text { sinq } \\
0 & \\
1 &
\end{array}\right]
$$

where $s$ and $q$ are known values that have been determined at the stage of local synthesis [2]. To determine the eccentric angle and the cradle angle, we use 
the following matrix equation

$$
\left[\begin{array}{cl}
s & \cos q \\
-s & \operatorname{sinq} \\
0 \\
1
\end{array}\right]=\left[\mathrm{H}_{02}^{(i i)}\right]\left[\begin{array}{l}
0 \\
0 \\
0 \\
1
\end{array}\right]
$$

where $\left[\begin{array}{llll}0 & 0 & 0 & 1\end{array}\right]$ are the coordinates of point $0_{2}^{(i)}$ in $\mathrm{s}_{2}^{(i)}$. Equations (3.2) and (2.7) yield

$$
\begin{aligned}
& s \cos q=\ell\left[\cos \phi_{c}-\cos \left(\phi_{c}+\phi_{e}\right)\right] \\
& s \sin q=\ell\left[\sin \phi_{c}-\sin \left(\phi_{c}+\phi_{e}\right)\right]
\end{aligned}
$$

where $\ell$ is a constant - the distance between the centers of the cradle and the eccentric. After transformations of equations (3.3) and (3.4), we obtain

$$
\begin{aligned}
& \frac{s}{2 l} \cos q=\sin \frac{\phi_{e}}{2} \sin \left(\phi_{c}+\frac{\phi_{e}}{2}\right) \\
& \frac{s}{2 l} \sin q=-\sin \frac{\phi_{e}}{2} \cos \left(\phi_{c}+\frac{\phi_{e}}{2}\right)
\end{aligned}
$$

Equations (3.5) and (3.6) provide in the area of $0<\phi_{e}<2 \pi, 0<\phi_{c}<2 \pi$ two solutions for $\phi_{e}$ and $\phi_{c}$ represented by the following equations

$$
\begin{aligned}
& \phi_{e}=2 \arcsin \frac{s}{2 \ell} \\
& \phi_{c}=q+90^{\circ}-\frac{\phi_{e}}{2}
\end{aligned}
$$

The two solutions are related as follows 


$$
\phi_{e}^{(2)}=2 \pi-\phi_{e}^{(1)} \phi_{c}^{(2)}=\phi_{c}^{(1)}+\phi_{e}^{(1)}-\pi
$$

The obtained results show that the required magnitude and orientation of vector $\mathbf{s}$ may be obtained by two combinations of parameters of machine-tool. settings, $\phi_{c}$ and $\phi_{e} \cdot$ Fig. 3.1 and Fig. 3.2 illustrate the installment of the cradle angle, $\phi_{c}$, and eccentric angle, $\phi_{e}$, for the cases where a left-hand pinion and a right-hand pinion are generated. In the practice only the first solution for the parameters $\phi_{e}$ and $\phi_{c}$ is applied (with $0<\phi_{e}<\pi$ ).

\section{Determination of Cutter Spindle Angle and Swivel Angle}

The combination of the cutter spindel angle, $\phi_{t}$, and the swivel angle, $\phi_{s}$, provides the required orientation of the unit vector $c$ of the tilted head cutter. The purpose of this section is to derive the equations that represent $\phi_{t}$ and $\phi_{S}$ in terms of the components of vector $\subseteq$. The eccentric angle, $\phi_{e}$, and the cradle angle, $\phi_{c}$, are considered as known at this stage of derivations. We will use for the derivation of the swivel angle two alternative techniques.

Pirst Technique Consider that vector $\subseteq$ is represented in $\mathrm{s}_{0}^{(i)}$ by the row matrix

$$
c_{0}=\left[\begin{array}{lll}
c_{x O} & c_{y 0} & c_{z 0}
\end{array}\right]=\left[\begin{array}{lll}
c_{x O} & c_{y 0} & \cos \mu
\end{array}\right]
$$

where $\mu$ is the angle that is formed by the cradle axis, $z_{0}^{(i)}$, and the axis of the tilted head cutter. The orientation of the axis of the tilted head-cutter is represented in $\mathrm{S}_{0}^{\left(i^{\prime}\right)}$ by the elements $a_{13}, a_{23}$, and $a_{33}$. Here: $a_{13}, a_{23}$, and $a_{33}$ are the direction cosines that are formed by axis $z_{4}^{(i)}$ (the headcutter axis) and the axes $x_{0}^{(i)}, y_{0}^{(i)}$ and $z_{0}^{(i)}$ (see matrix (2.5)). 
The cutter spindle angle can be determined from the equation

$$
a_{33}=\cos \mu
$$

Equating equation (3.11) and using the expression for a 33 (see equations $(2.6))$, we obtain

$$
\sin ^{2} \varepsilon \cos \phi_{t}+\cos ^{2} \varepsilon=\cos \psi
$$

Equation (3.11) yields

$$
\sin \frac{\varphi_{t}}{2}=\frac{\sin \frac{\mu}{2}}{\sin \varepsilon}
$$

This equation provides two solutions for the cutter spindle angle, $\phi_{t}$, considering that the magnitude of $\mu$ is given. The two solutions for $\phi_{t}$ are related as follows

$$
\phi_{t}^{(2)}=2 \pi-\phi_{t}^{(1)}
$$

In the practice only the first solution for $\phi_{t}^{(1)}\left(0<\phi_{t}^{(1)}<\pi\right)$ is used. The derivation of the swivel angle is based on equations

$$
a_{13}=c_{x o}, a_{23}=c_{y o}
$$

Using the expressions for $a_{13}$ and $a_{23}$ (see equations (2.6)), we obtain

$$
\frac{\cos \tau \sin 2 \varepsilon}{2}\left(1-\cos \phi_{t}\right)+\sin \tau \sin \varepsilon \sin \phi_{t}=c_{x 0}
$$




$$
-\frac{\sin \tau \sin 2 \varepsilon}{2}\left(1-\cos \phi_{t}\right)+\cos \tau \sin \varepsilon \sin \phi_{t}=c_{y o}
$$

where $\tau=\dot{\phi}_{c}+\phi_{e}+\phi_{s} \cdot$

We may transform equations (3.16) and (3.17) and represent them as a system of two pseudo-linear equations in the unknowns $\cos t$ and $\sin \tau$ as follows

$$
\begin{array}{r}
a_{11} \cos \tau+a_{12} \sin \tau=b_{1} \\
a_{21} \cos \tau-a_{22} \sin \tau=b_{2}
\end{array}
$$

Here:

$$
\begin{aligned}
& a_{11}=a_{22}=\sin 2 \varepsilon \sin ^{2} \frac{\phi_{t}}{2} ; a_{12}=a_{21}=\sin \varepsilon \sin \phi_{t} ; \\
& b_{1}=c_{x o}, b_{2}=c_{\text {yo }}
\end{aligned}
$$

The solution of equation system (3.18) for the unknowns is

$$
\begin{aligned}
& \cos \tau=\frac{a_{11} b_{1}+a_{12} b_{2}}{a_{11}^{2}+a_{12}^{2}}=A \\
& \sin \tau=\frac{a_{21} b_{1}-a_{11} b_{2}}{a_{11}^{2}+a_{12}^{2}}=B
\end{aligned}
$$

or

$$
\tan \frac{\tau}{2}=\frac{1-A}{B}
$$

Then we obtain that 


$$
\phi_{s}=\tau-\phi_{c}-\phi_{e}
$$

\section{Second Technique}

Consider that vector $\subseteq$ is represented in coordinate system $s_{2}^{(i)}$ that is rigidly connected to the eccentric and is given by the row matrix

$$
\left[c_{2}\right]=\left[\begin{array}{lll}
c_{x 2} & c_{y 2} & c_{z 2}
\end{array}\right]
$$

Here:

$$
\left[c_{2}\right]=\left[L_{20}^{(i i)}\right]\left[c_{0}\right]
$$

where $\left[\mathrm{L}_{20}^{(\mathrm{ii})}\right]$ represents the coordinate transformation from $\mathrm{s}_{0}^{(i)}$ to $\mathrm{s}_{2}^{(i)}$ and is given by (see equation (2.7))

$$
\begin{aligned}
& {\left[\mathrm{L}_{20}^{(\mathrm{ii})}\right]=\left[\mathrm{L}_{02}^{(\mathrm{ii})}\right]^{\mathrm{T}}} \\
& =\left[\begin{array}{ccc}
\cos \left(\phi_{c}+\phi_{e}\right) & -\sin \left(\phi_{c}+\phi_{e}\right) & 0 \\
\sin \left(\phi_{c}+\phi_{e}\right) & \cos \left(\phi_{c}+\phi_{e}\right) & 0 \\
0 & 0 & 1
\end{array}\right]
\end{aligned}
$$

The unit vector of the axis of tilted head-cutter is represented in $S_{2}^{(i)}$ by its direction cosines $b_{13}, b_{23}$ and $b_{33}$ (see matrix (2.9)). The determination of the cutter spindle angle, $\phi_{t}$, and the swivel angle, $\phi_{s}$, is base.t on the following equations

$$
b_{13}=c_{x 2}, \quad b_{23}=c_{y 2}, \quad b_{33}=c_{z 2}
$$


Equation $b_{33}=c_{z 2}$ provides the same solution for $\phi_{t}$ as it has been represented by equation (3.12). Using the remaining two equations

$$
b_{13}=c_{x 2}, \quad b_{23}=c_{y 2}
$$

we obtain after transformations the following system of two pseudo-linear equations in the unknowns $\cos \phi_{S}$ and $\sin \phi_{S}$

$$
\begin{aligned}
& m_{11} \cos \phi_{s}+m_{12} \sin \phi_{s}=n_{1} \\
& m_{21} \cos \phi_{s}+m_{22} \sin \phi_{s}=n_{2}
\end{aligned}
$$

Here:

$$
\begin{aligned}
& \mathrm{m}_{11}=\frac{\sin 2 \varepsilon}{2}\left(1-\cos \phi_{t}\right), \quad \mathrm{m}_{12}=\sin \varepsilon \sin \phi_{t} \\
& \mathrm{~m}_{21}=\mathrm{m}_{12}, \quad \mathrm{~m}_{22}=-\mathrm{m}_{11}, \quad \mathrm{n}_{1}=c_{x 2}, \quad \mathrm{n}_{2}=c_{y 2}
\end{aligned}
$$

The solution of equations (3.25) and (3.26) for the unknowns cos $\phi_{s}$ and $\sin \phi_{s}$ yields

$$
\begin{aligned}
& \cos \phi_{s}=\frac{m_{11} n_{1}+m_{12} n_{2}}{m_{11}^{2}+m_{12}^{2}}=C \\
& \sin \phi_{s}=\frac{m_{12} n_{1}-m_{11} n_{2}}{m_{11}^{2}+m_{12}^{2}}=D
\end{aligned}
$$

where $m_{11}^{2}+m_{12}^{2}=4 \sin ^{2} \varepsilon \sin ^{2} \frac{\phi_{t}}{2} \cos ^{2} \frac{\mu}{2}$ 
or

$$
\tan \frac{\phi_{s}}{2}=\frac{1-C}{D}
$$

\section{Numerical Example}

The input data in the discussed example is adopted from literature [2] and is represented in coordinate system $\mathrm{S}_{0}^{(i)}$ as follows (length units in $\mathrm{mm}$ ):

$$
\begin{aligned}
& \underline{s}=\left[133.09 \cos \left(84.033^{\circ}\right) ;-133.09 \sin \left(84.033^{\circ}\right) ; 0\right] \\
& \underline{s}=[0.1085 ;-0.1157 ; 0.9874] ; \ell=222.25 ; \varepsilon=15^{0} \\
& \cos \eta=c_{2}=0.9874
\end{aligned}
$$

The following computations has been performed for the Gleason Cutting Machine. \#116. Equations (3.7) and (3.8) provide the data for the eccentric angle, $\phi_{\ell}$, and the cradle angle, $\phi_{C}$. Equation (3.13) provides the data for the cutter spinde angle, $\phi_{t}$. Equations (3.21), (3.20) and (3.19) provide the data for the swivel angle, $\phi_{s}$. Alternative equations (3.27) and (3.28) for determination of $\phi_{S}$ may be also used.

The final results of computations are represented in the following table

\section{TABLE 1}

\begin{tabular}{|l|c|c|c|}
\hline $\begin{array}{c}\text { Eccentric } \\
\text { Angle, } \phi_{\mathrm{e}}\end{array}$ & $\begin{array}{c}\text { Cradle Angle, } \\
\phi_{\mathrm{c}}\end{array}$ & $\begin{array}{c}\text { Cutter } \\
\text { Spindle Angle, } \\
\phi_{\mathrm{t}}\end{array}$ & $\begin{array}{c}\text { Swivel } \\
\text { Angle, } \\
\phi_{\mathrm{s}}\end{array}$ \\
\hline $73.57^{\circ}$ & $137.25^{\circ}$ & $35.7175^{\circ}$ & $268.10^{\circ}$ \\
\hline
\end{tabular}




\section{Conclusion}

The kinematics of the Gleason's mechanism for the installment of the machine-tool settings has been investigated.

The basic euations for the determination of the eccentric angle, cradle angle, cutter spindle angle and the swivel angle for the gear cutting machine have been developed. These equations provide the required magnitude and orientation of position-vector $s$ and the orientation of unit vector $c$ of the axis of the tilted head-cutter axis (Fig. $1.1, b$ ).

A numerical example that illustrates the proposed computation procedure has been represented. 


\section{Nomenclature}

$\subseteq$ Unit vector of tilted head-cutter axis

\& Machine constant

$P \quad$ Angle determining the projection of cutter axis on machine plane

q Basic cradle angle determining location of cutter axis

s Radial setting for pinion head-cutter axis

$\mu \quad$ Angle of head-cutter axis inclined with cradle axis

$\varepsilon \quad$ Wedge angle

$\phi_{c}$ Cradle angle

$\phi_{\ell} \quad$ Eccentric angle

$\phi_{S}$ Swivel angle

$\phi_{t} \quad$ Cutter spindle angle 


\section{References}

1. Gleason Works, 1981, "Understanding Tooth Contact Analysis", Rochester, N.Y., 14692, Publication No. SD3139.

2. Litvin, F.L., and Gutman, Y.I., 1981, Methods of Synthesis and Analysis for Hypoid-Gear Drives of "Formate" and "Helix-form", J. of Mechanical Design, Vol. 103, pp. 89-113.

3. Lehmann, E.P., 1977, Tilt Generation of Spiral Bevel and Hypoid Gears, Gleason Works. 
(a)

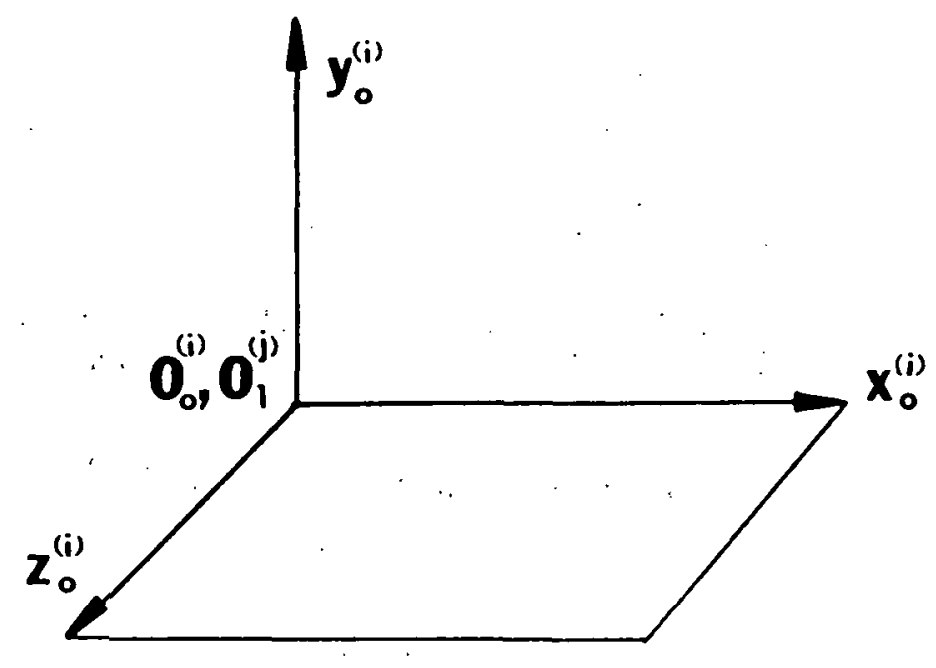

(b)

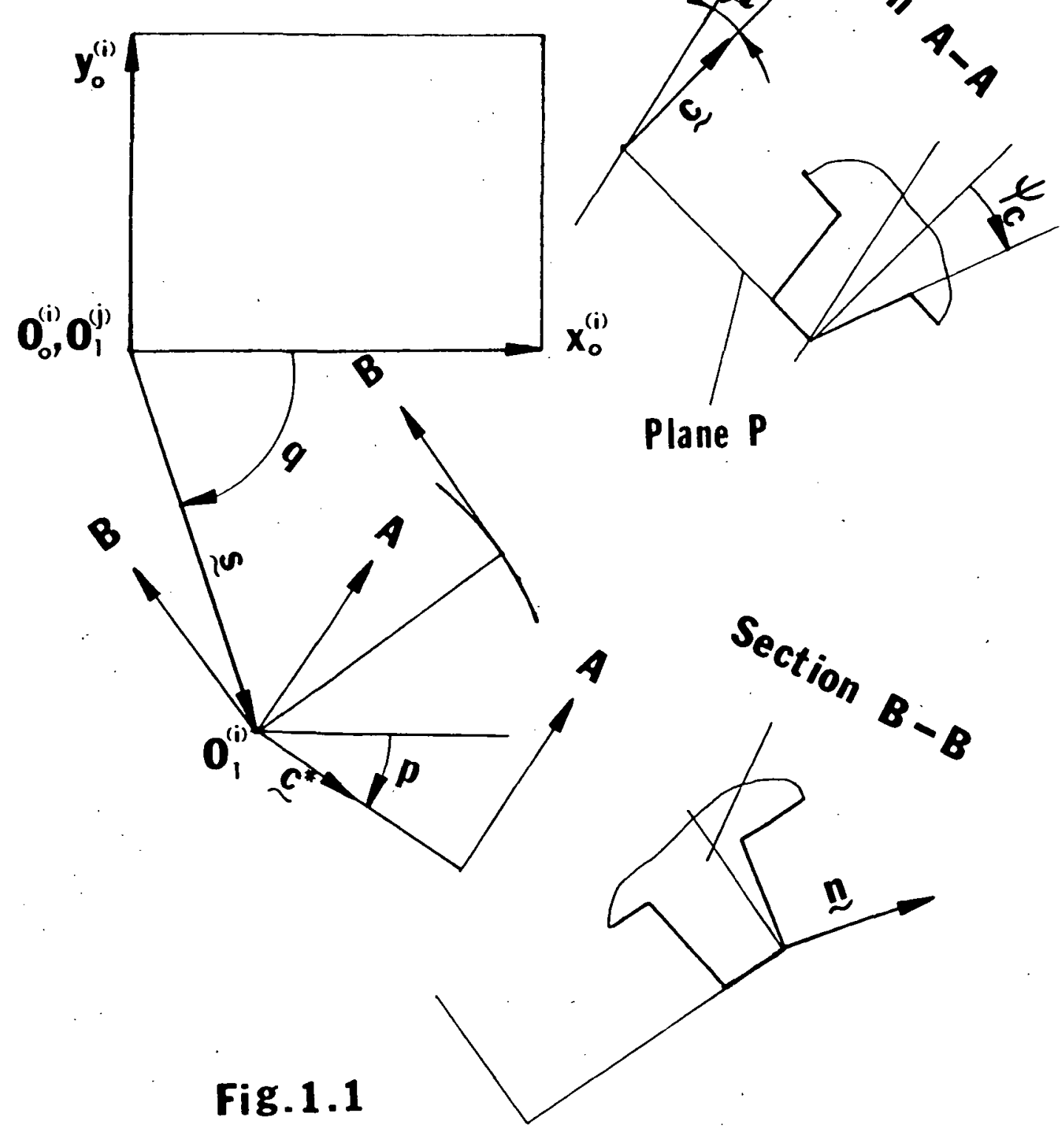

Basic Coordinate System 


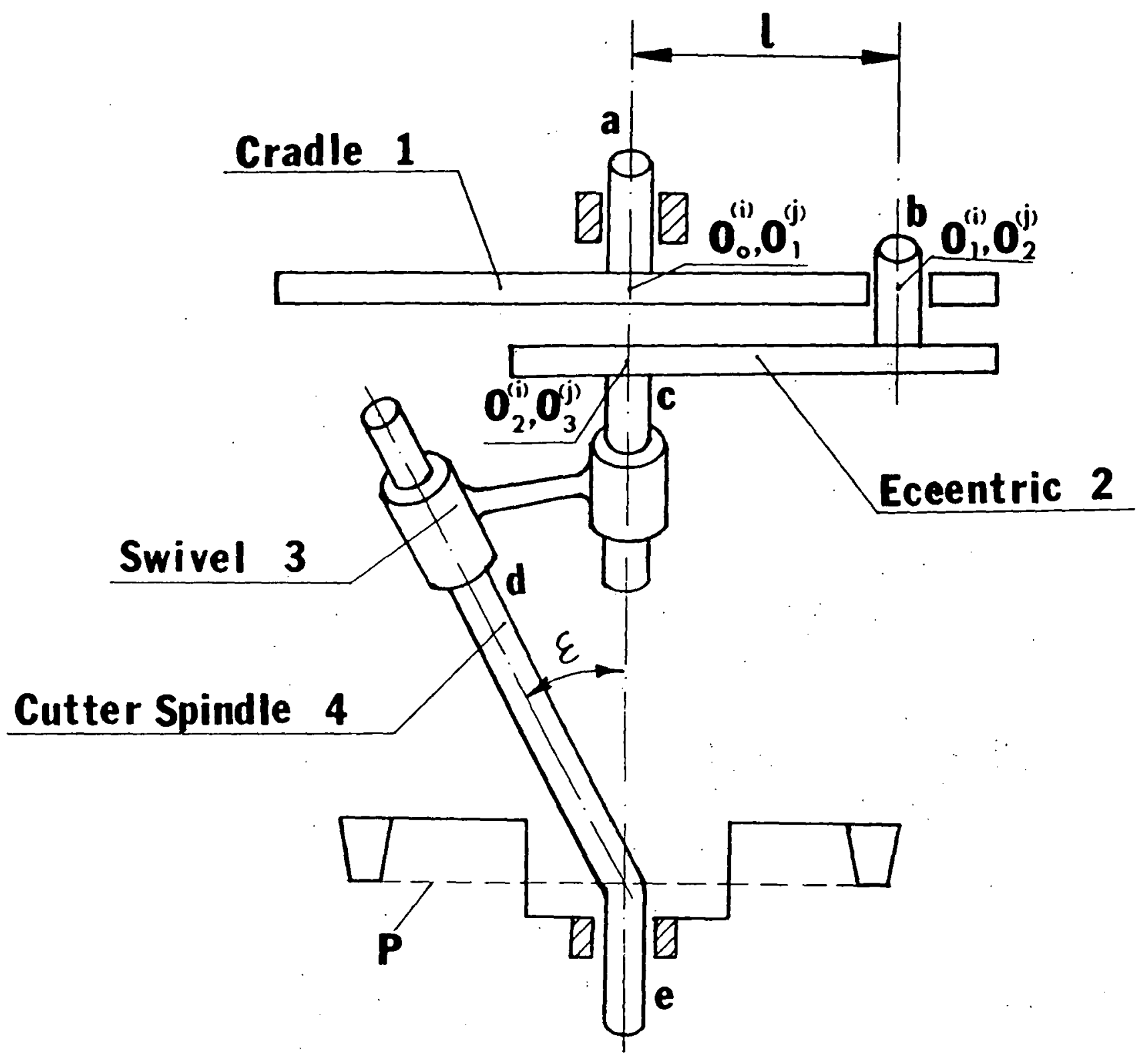

Fig.1.2

Installment Mechanism 


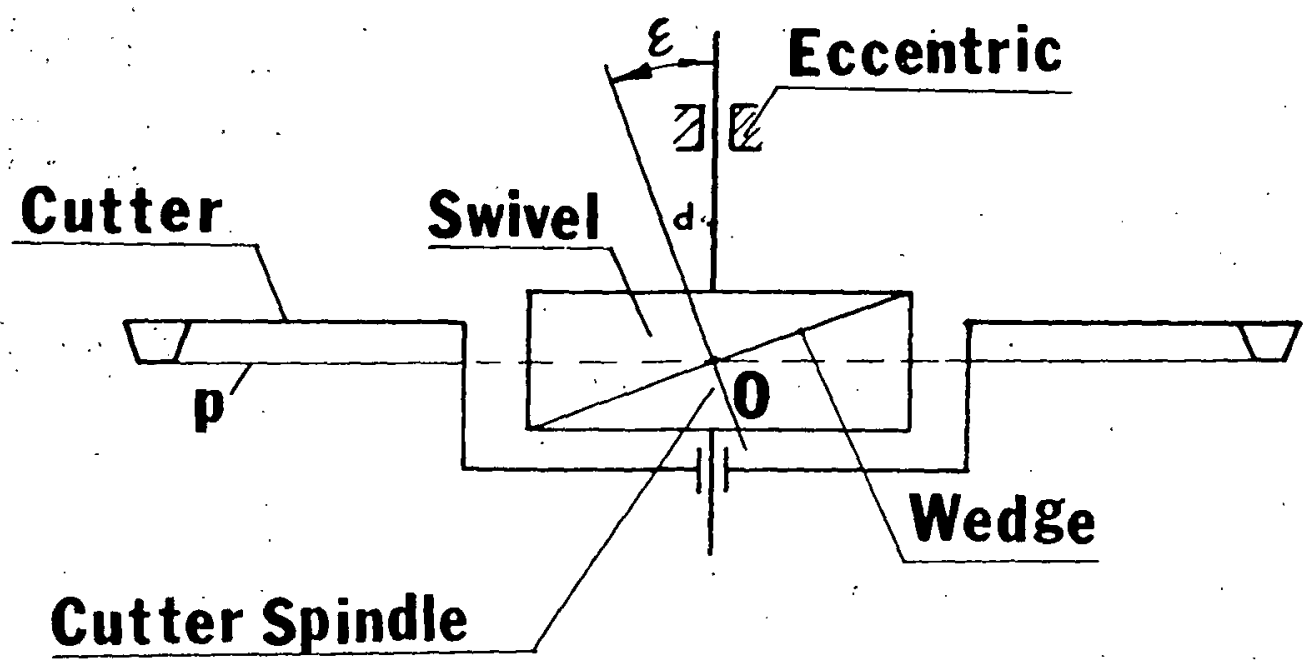

Fig. 1.3

Tilt Mechanism 


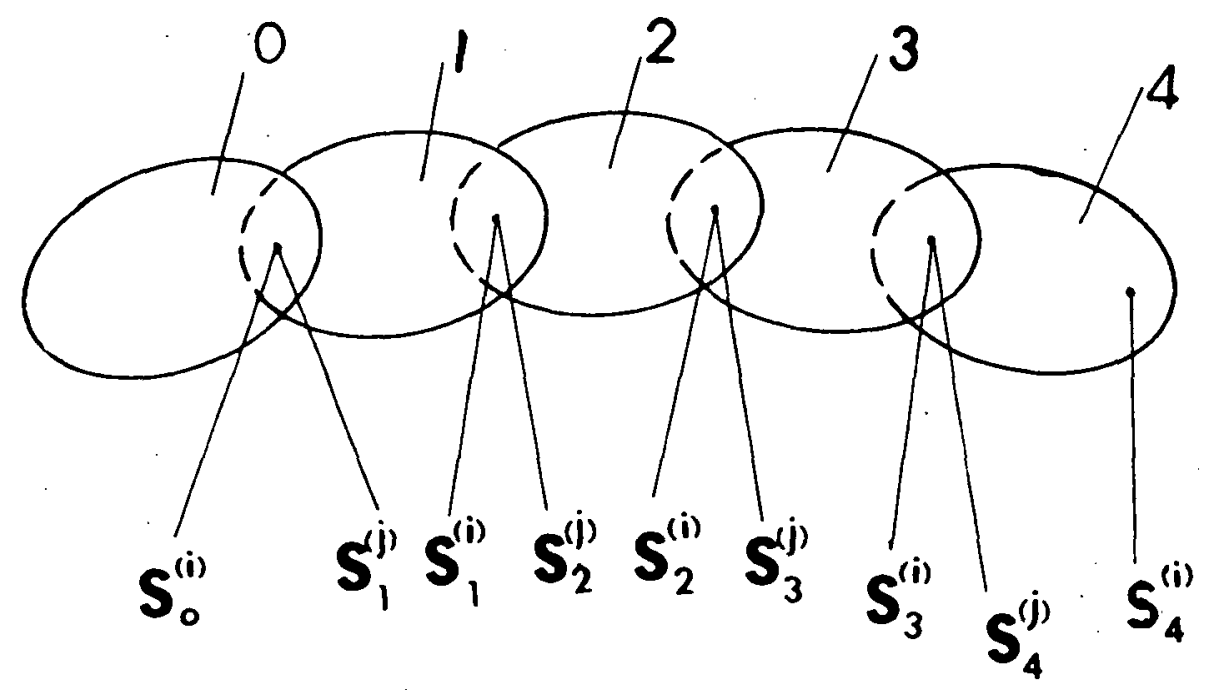

Fig. 2.1

\section{Applied Coordinate Systems}


(a)

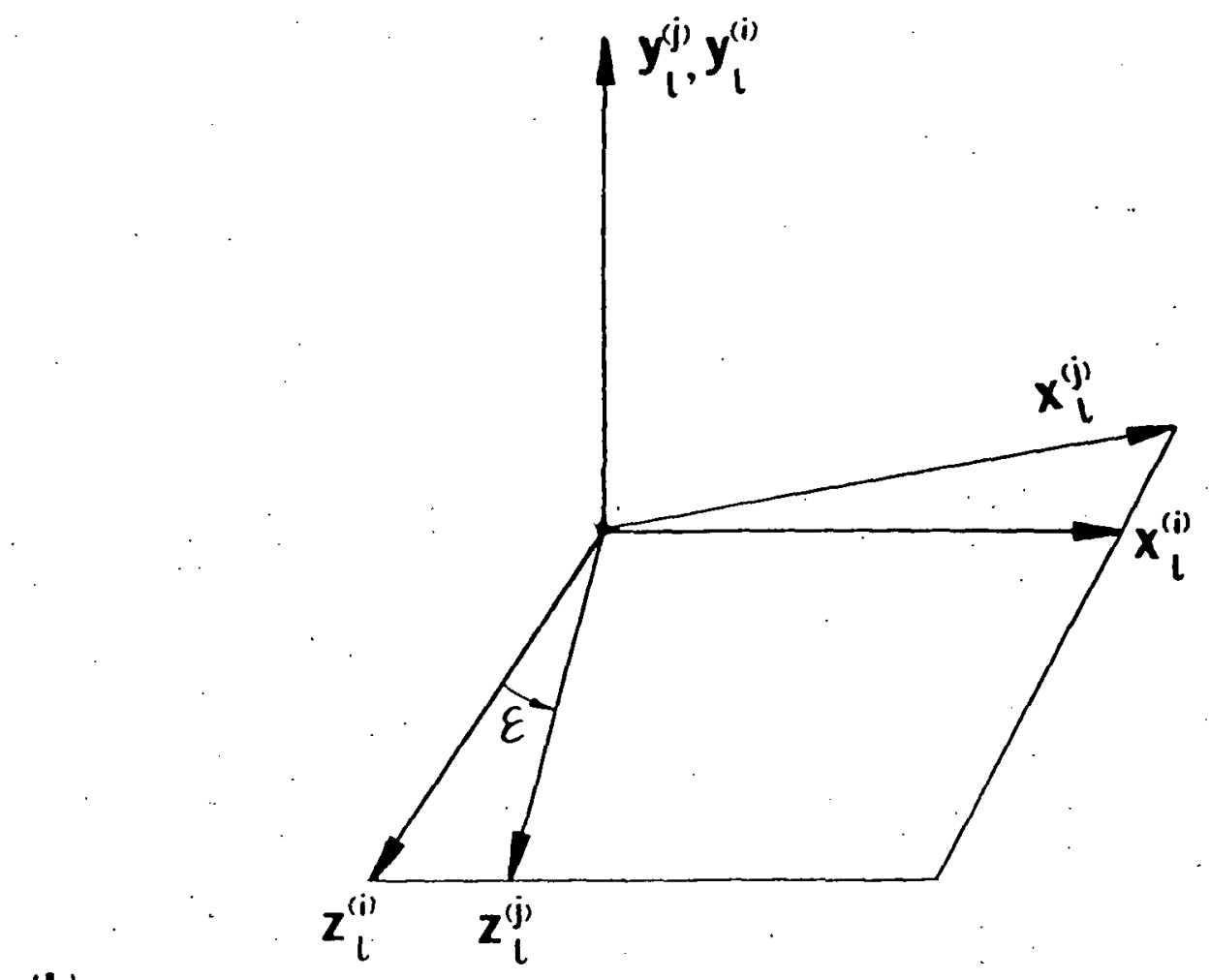

(b)

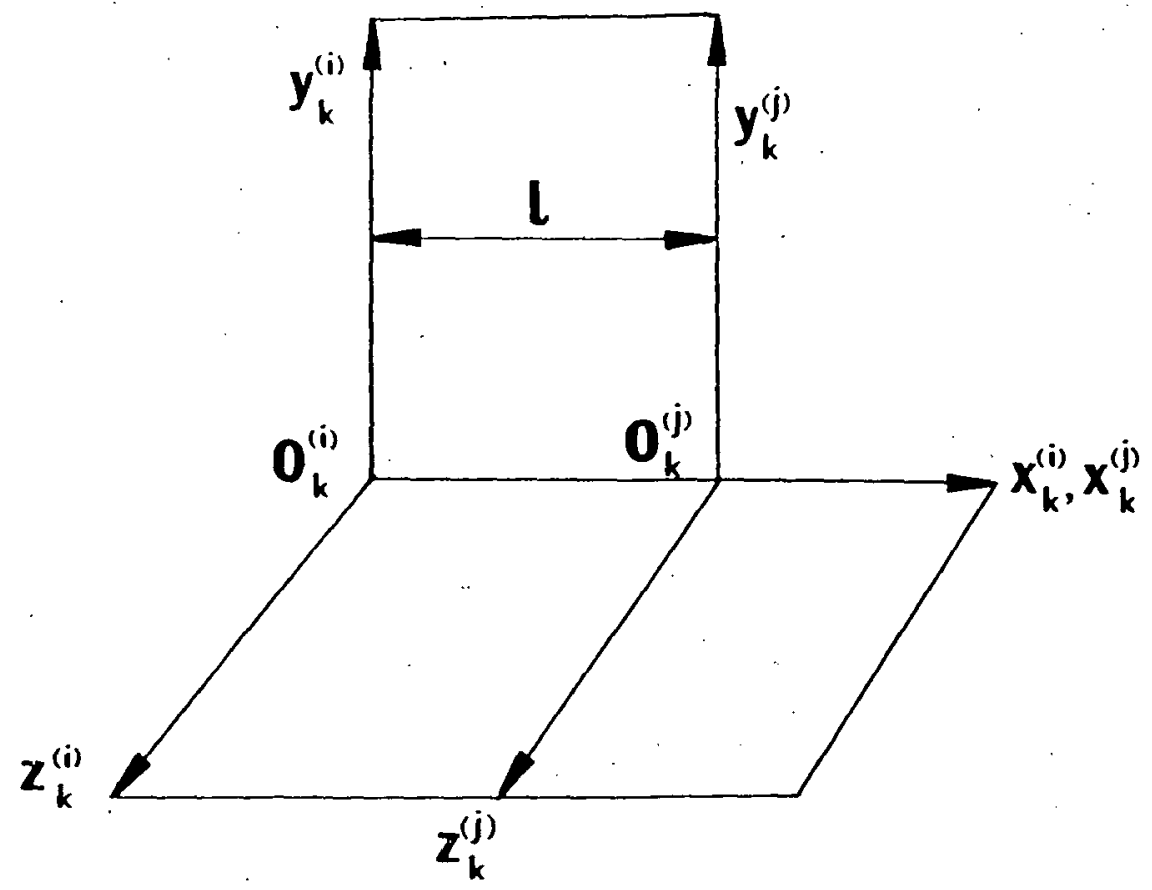

Fig. 2.2

Coordinate Iransformation by Geometry Matrix 


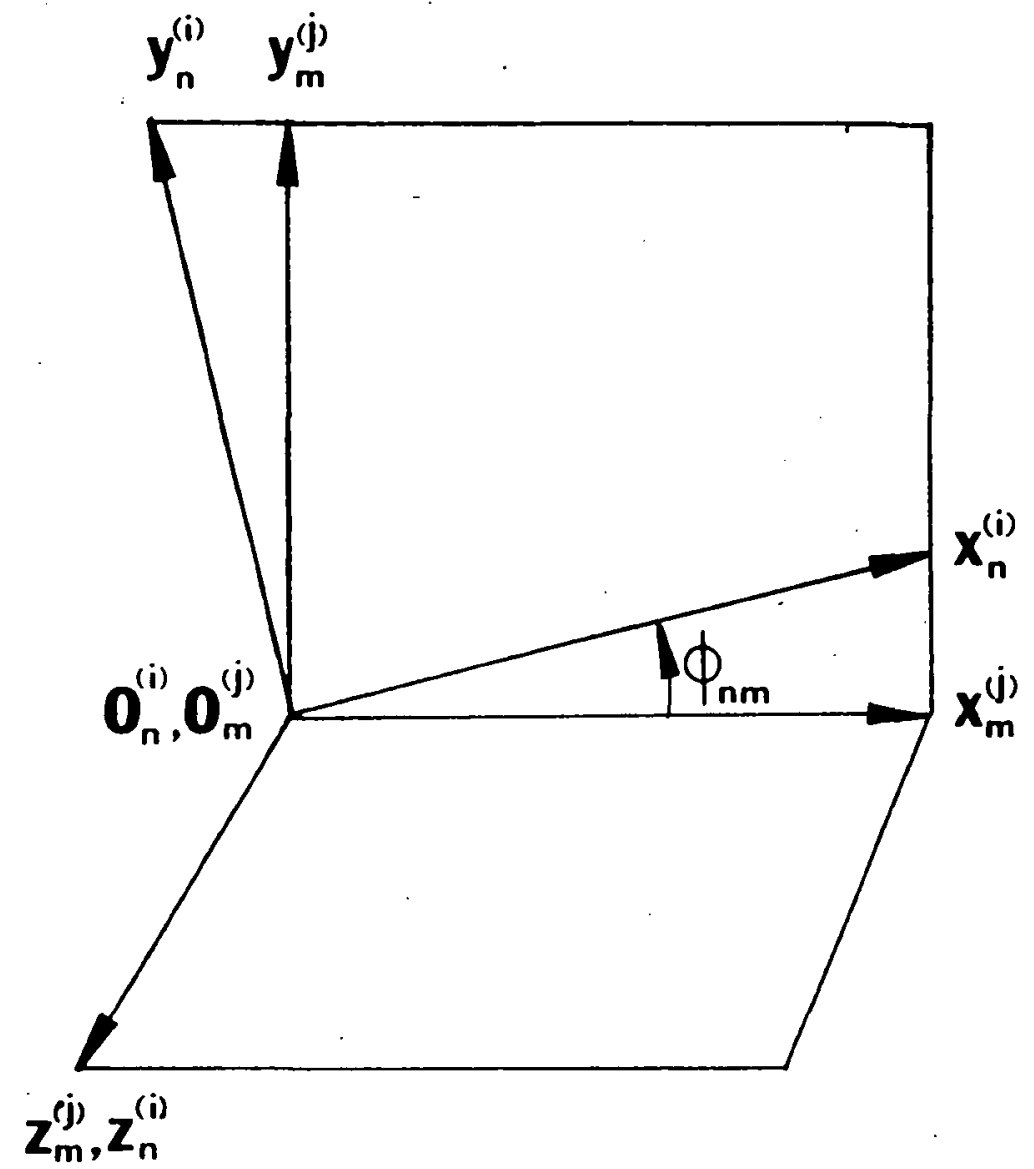

Fig.2.3

Coordinate Transformation by Rotation Matrix 
(a)

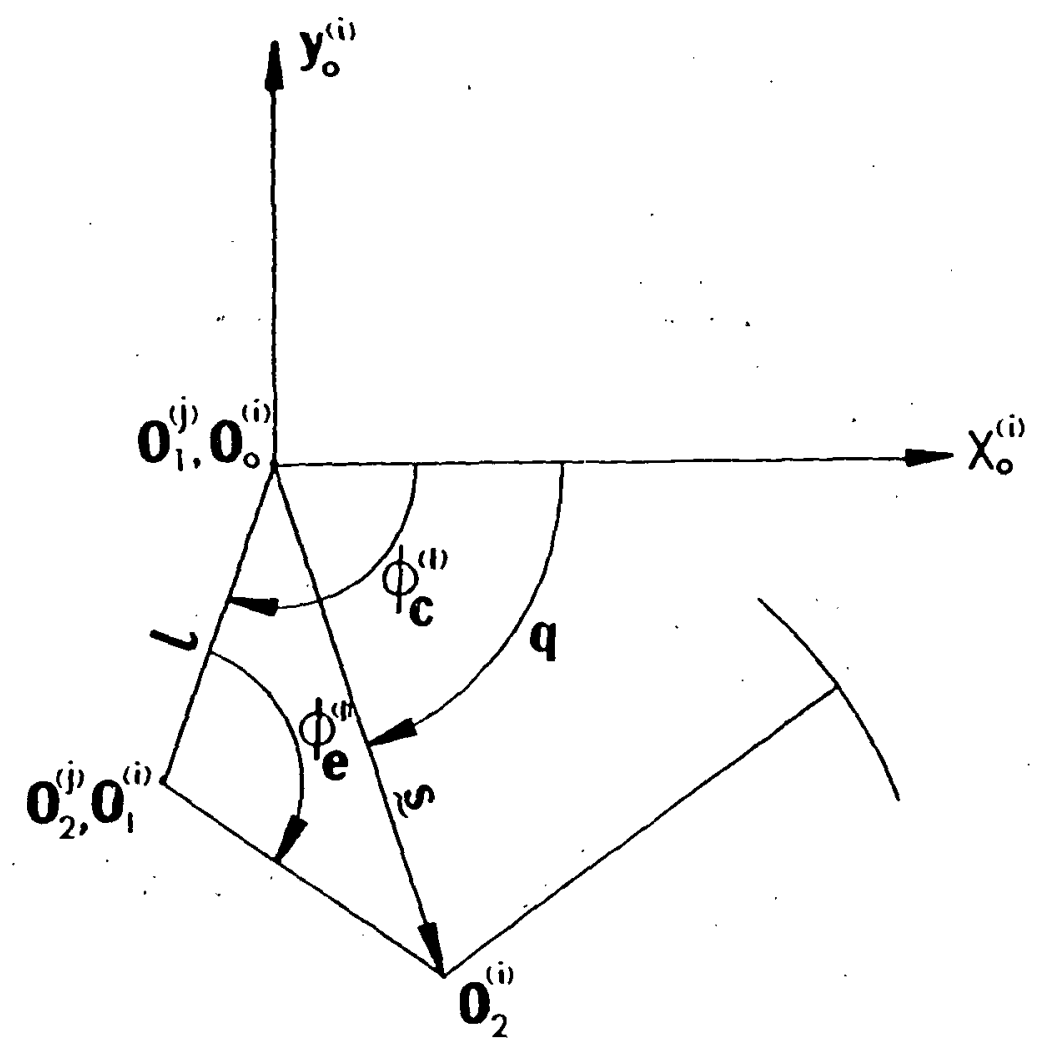

(b)

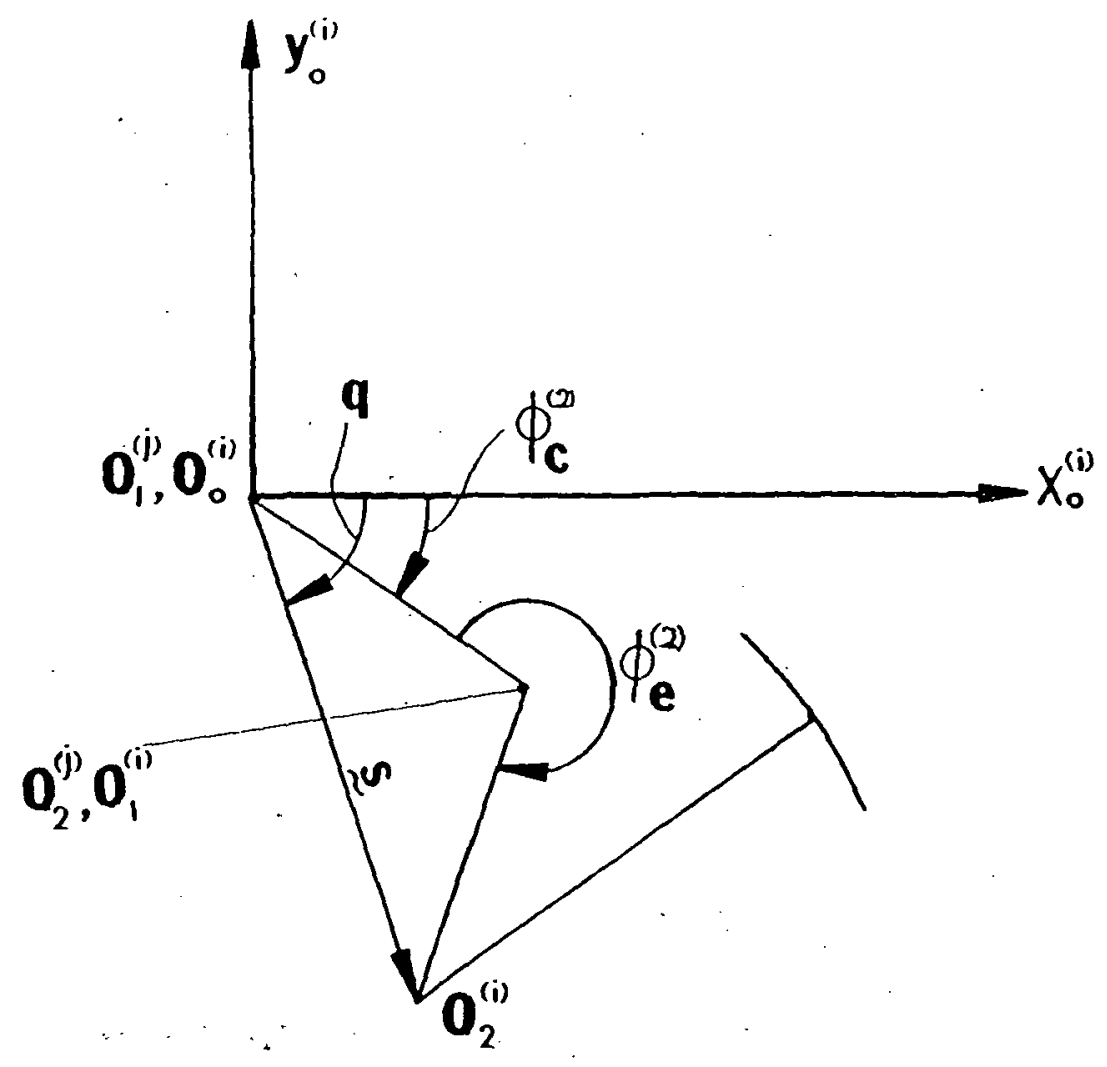

Fig. 3.1

Basic Settings for Left-Hand Pinion 
(a)

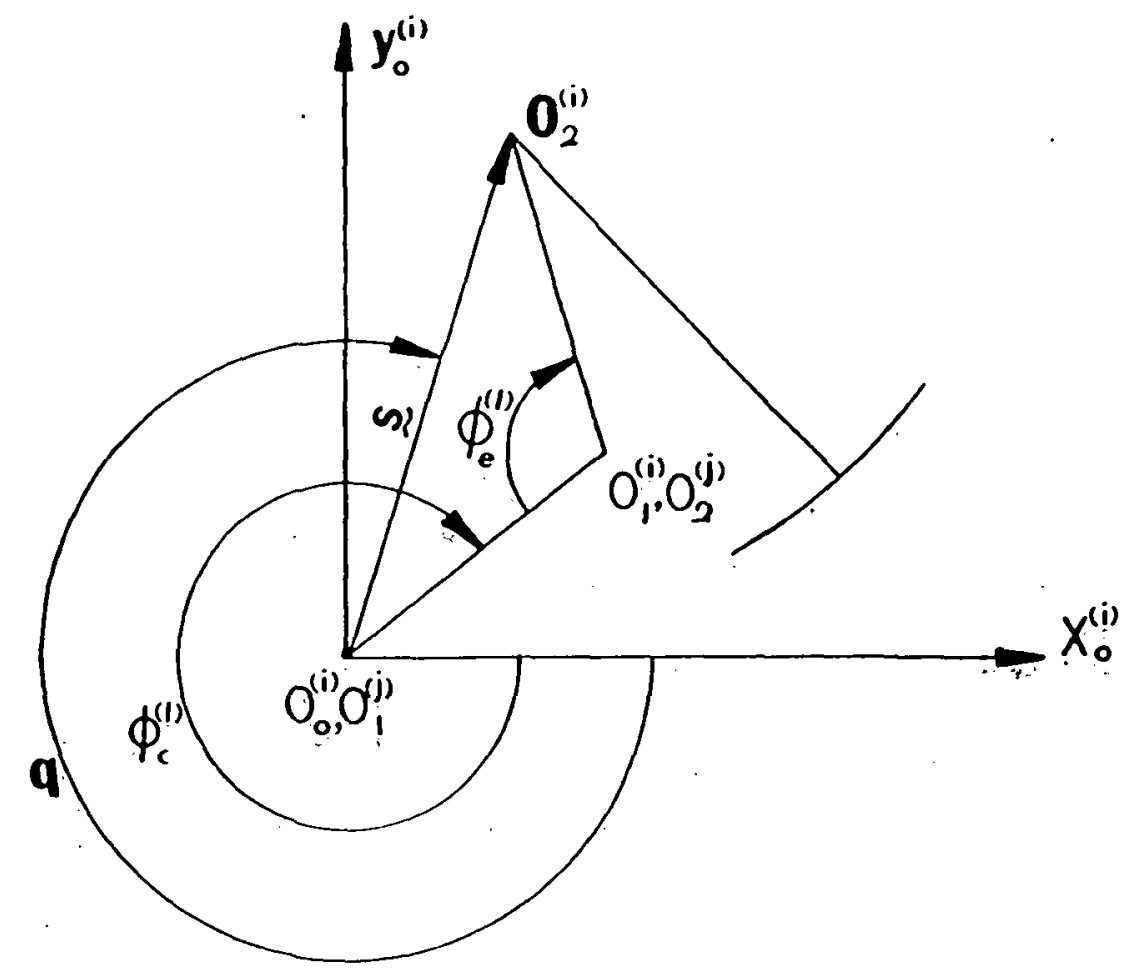

(b)

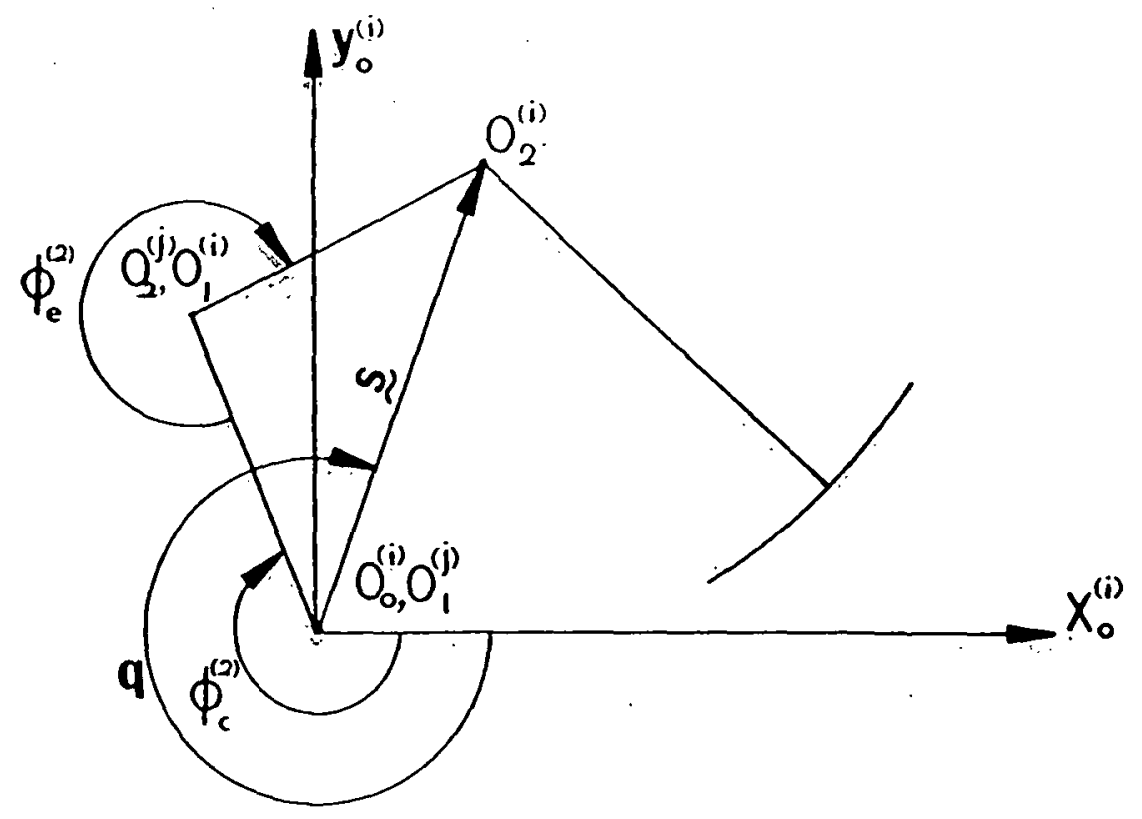

Fig. 3.2

Basic Settings for Right-Hand Pinion 


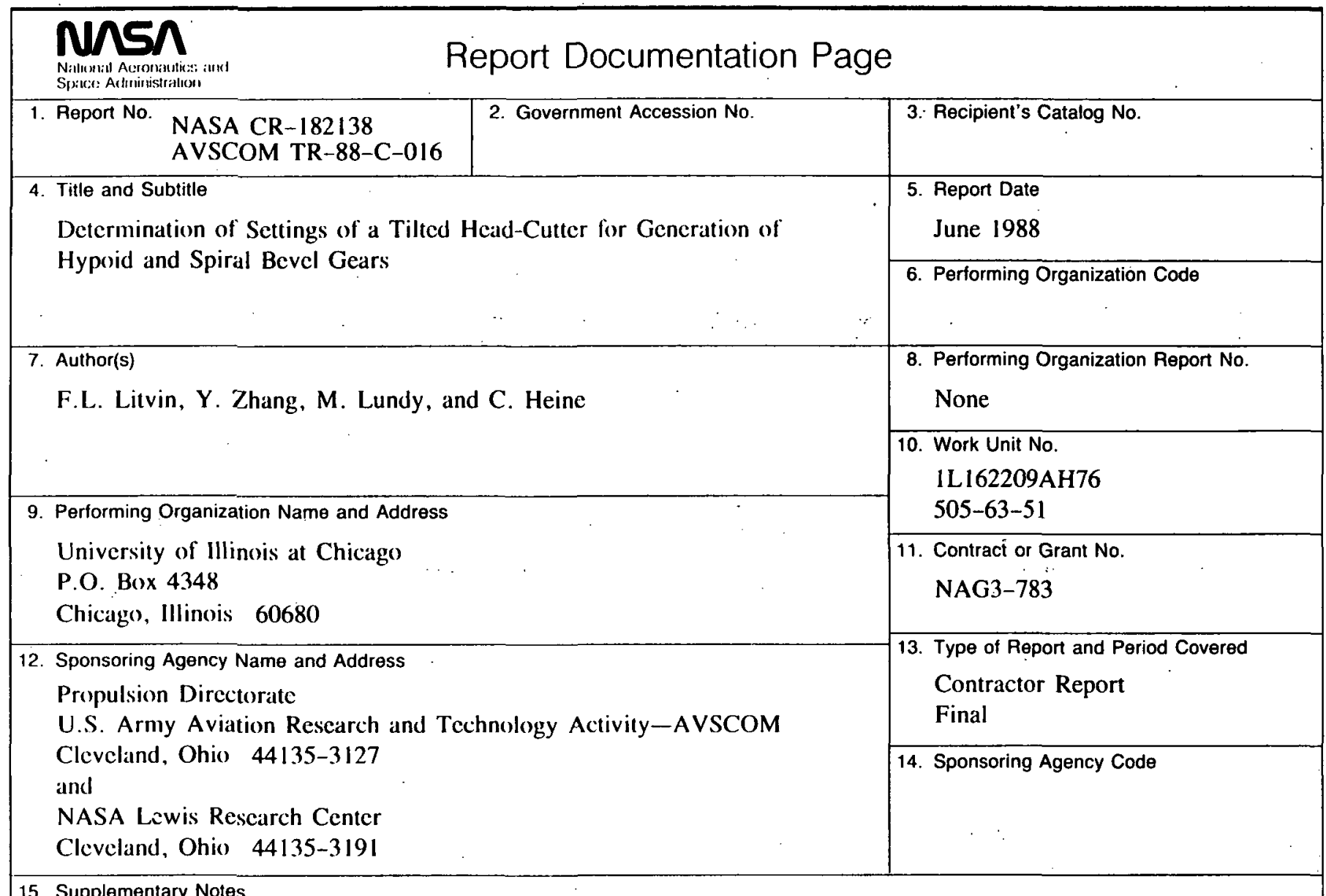

15. Supplementary Notes

Project Manager, Robert F. Handschuh, Propulsion Directorate, U.S. Army Aviation Research and Technology Aclivity-AVSCOM, Lewis Research Center. F.L. Litvin and Y. Zhang, University of Illinois at Chicago; M. Lundy and C. Heine, Dana Corporation, Fort Wayne, Indiana.

16. Abstract

Kinematics of Gleason mechanisms of hypoid and spiral bevel cutting machines is considered. These mechanisms are designated to install the position and tilt of the head cutter. The tilt of the head cutter with standard blades provides the required pressure angle. The authors have developed the matrix presentation of kinematics of these mechanisms and basic equations for the required settings. An example is presented based on the developed computation procedure.

17. Key Words (Suggested by Author(s))

Hypoid gears

Spiral bevel gears

Gear manufacture

Gcars
18. Distribution Statement

Unclassified-Unlimited

Subject Category 37

19. Security Classif. (of this report)

Unclassified

20. Security Classif. (of this page)

Unclassified
21. No of pages

26
22. Price" A03 